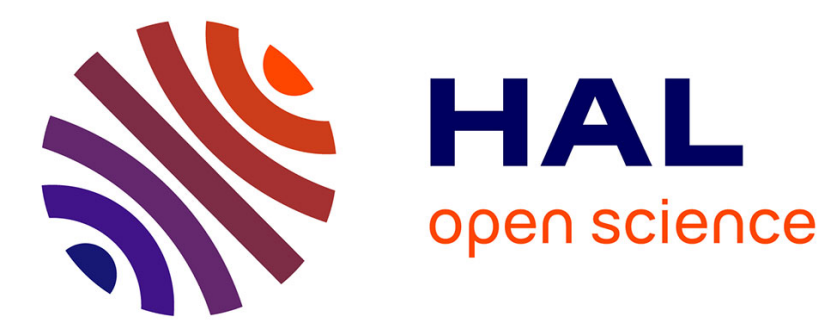

\title{
Computer Algebra in the Service of Enumerative Combinatorics
}

\author{
Alin Bostan
}

\section{To cite this version:}

Alin Bostan. Computer Algebra in the Service of Enumerative Combinatorics. International Symposium on Symbolic and Algebraic Computation (ISSAC), Jul 2021, Saint Petersburg, Russia. pp. 1-8, 10.1145/3452143.3465507 . hal-03427873

\section{HAL Id: hal-03427873 https://hal.science/hal-03427873}

Submitted on 14 Nov 2021

HAL is a multi-disciplinary open access archive for the deposit and dissemination of scientific research documents, whether they are published or not. The documents may come from teaching and research institutions in France or abroad, or from public or private research centers.
L'archive ouverte pluridisciplinaire HAL, est destinée au dépôt et à la diffusion de documents scientifiques de niveau recherche, publiés ou non, émanant des établissements d'enseignement et de recherche français ou étrangers, des laboratoires publics ou privés. 


\title{
Computer Algebra in the Service of Enumerative Combinatorics
}

\author{
Alin Bostan \\ Inria, Université Paris-Saclay, France \\ alin.bostan@inria.fr
}

\begin{abstract}
Classifying lattice walks in restricted lattices is an important problem in enumerative combinatorics. Recently, computer algebra has been used to explore and to solve a number of difficult questions related to lattice walks. We give an overview of recent results on structural properties (e.g., algebraicity versus transcendence) and on explicit formulas for generating functions of walks with small steps in the quarter plane. In doing so, we emphasize the algorithmic nature of the methodology, especially two important paradigms: "guess-and-prove" and "creative telescoping".
\end{abstract}

\section{CCS CONCEPTS}

- Computing methodologies $\rightarrow$ Algebraic algorithms.

\section{KEYWORDS}

Computer algebra; Experimental mathematics; Guess-and-Prove; Creative telescoping; Enumerative combinatorics; Lattice paths; Generating functions; D-finite functions; Algebraic functions.

ACM Reference Format:

Alin Bostan. 2021. Computer Algebra in the Service of Enumerative Combinatorics. In Proceedings of the 2021 International Symposium on Symbolic and Algebraic Computation (ISSAC '21), July 18-23, 2021, Virtual Event, Russian Federation. ACM, New York, NY, USA, 8 pages.

\section{GENERAL PRESENTATION}

\subsection{Prelude}

Consider the following innocent-looking problem.

A tandem-walk is a path in $\mathbb{Z}^{2}$ taking steps from $\{\uparrow, \leftarrow, \searrow\}$ only. Show that, for any integer $n \geq 0$, the following quantities are equal:

(i) the number $a_{n}$ of tandem-walks of length $n$ (i.e., using $n$ steps), confined to the upper half-plane $\mathbb{Z} \times \mathbb{N}$, that start and end at $(0,0)$;

(ii) the number $b_{n}$ of tandem-walks of length $n$ confined to the quarter plane $\mathbb{N}^{2}$, that start at $(0,0)$ and finish on the diagonal $x=y$.

For instance, when $n=3$, this common value is $a_{3}=b_{3}=3$, as shown in the next picture, obtained by exhaustive enumeration.

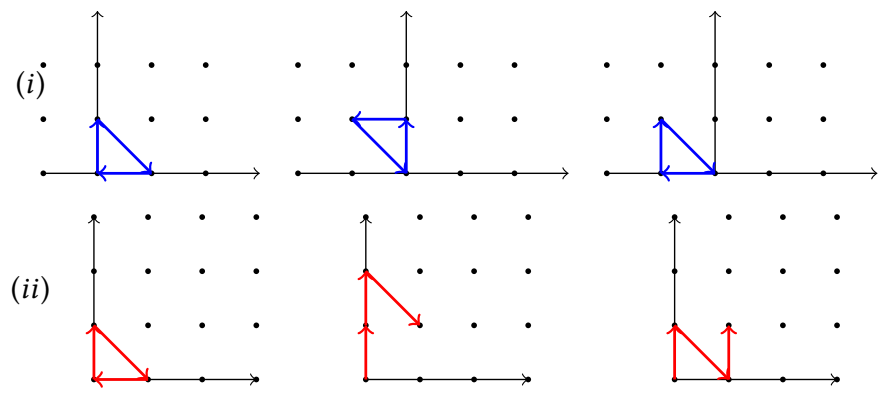

Preprint to appear in ISSAC '21, July 18-23, 2021, Virtual Event, Russian Federation. 2021.
It appears that this problem is far from being trivial. Several solutions exist, but none of them is elementary. One of the main aims of the present text is to convince the reader that this problem (and many others with a similar flavor) can be solved with the help of a computer. More precisely, computer algebra tools can be used to discover and to prove the following equalities

$$
a_{3 n}=b_{3 n}=\frac{(3 n) !}{n !^{2} \cdot(n+1) !} \quad \text { and } \quad a_{m}=b_{m}=0 \quad \text { if } 3 \nmid m .
$$

It goes without saying that such a simple and beautiful expression cannot be the result of mere chance. It turns out that closed forms are quite rare for this kind of enumeration problem. Nevertheless, even in the absence of nice formulas, the structural properties of the corresponding enumeration sequences reflect the symmetries of the step set and of the evolution domain. Equation (1) shows that the sequences $\left(a_{n}\right)$ and $\left(b_{n}\right)$ are P-recursive, that is, they satisfy a linear recurrence with polynomial coefficients (in the index $n$ ). Equivalently, their generating functions $\sum_{n \geq 0} a_{n} t^{n}$ and $\sum_{n \geq 0} b_{n} t^{n}$ are D-finite, that is, they satisfy linear differential equations with polynomial coefficients (in the variable $t$ ). On the methodological (i.e., computer-algebraic) side, one of the main messages that will emerge from the text is that, in the absence of closed formulas, the (recurrence/differential) equations themselves constitute the appropriate data structure to represent and manipulate P-recursive sequences and D-finite functions [65]. On the application (i.e., combinatorial) side, the main message is that these important properties of the enumeration sequences are intimately related to the finiteness of a certain group, naturally attached to the step set $\{\uparrow, \leftarrow, \searrow\}$.

\subsection{General context: walks confined to cones}

Let us put the previous problem into a more general framework. Let $d \geq 1$ be an integer (dimension), let $\mathscr{S}$ be a finite subset (called step set, or model) of vectors in $\mathbb{Z}^{d}$, and $p_{0} \in \mathbb{Z}^{d}$ (starting point). An $\mathscr{S}$-path (or, $\mathscr{S}$-walk) of length $n$ starting at $p_{0}$, is a sequence $\left(p_{0}, p_{1}, \ldots, p_{n}\right)$ of elements in the lattice $\mathbb{Z}^{d}$ such that $p_{i+1}-p_{i} \in \mathscr{S}$ for all $0 \leq i<n$. Let $\mathscr{C}$ be a cone of $\mathbb{R}^{d}$, that is, a subset of $\mathbb{R}^{d}$ such that $r \cdot v \in \mathscr{C}$ for any $v \in \mathscr{C}$ and $r \geq 0$, assumed to contain $p_{0}$. In combinatorics and in probability theory, one is interested in the (exact or asymptotic) enumeration of $\mathscr{S}$-walks confined to the cone $\mathscr{C}$, potentially subject to additional constraints.

Example 1.1. Consider the model $\mathscr{S}=\{\rightarrow, \leftarrow, \nearrow, \swarrow\}$, that is $\mathscr{S}=\{(1,0),(-1,0),(1,1),(-1,-1)\}$ (called the Gessel model) in dimension $d=2$, with starting point $p_{0}=(0,0)$ and cone $\mathscr{C}=\mathbb{R}_{\geq 0}^{2}$ (the quarter plane). Figure 1 displays the model $\mathscr{S}$ (on the left), and an $\mathscr{S}$-walk of length $n=2178$ confined to $\mathscr{C}$ (on the right).

The main typical questions in this context are the following:

- What is the number $a_{n}$ of $n$-step $\mathscr{S}$-walks contained in $\mathscr{C}$ ?

- For $\mathbf{i} \in \mathscr{C}$, what is the number $a_{n ; \mathbf{i}}$ of such walks ending at i? 

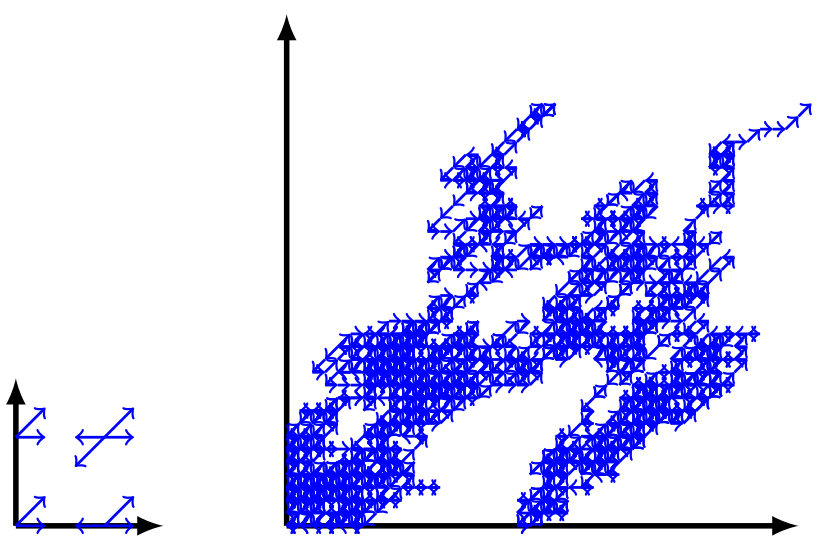

Figure 1: Gessel walks: the allowed steps, and a random walk

- What is the nature of their generating functions

$$
A(t)=\sum_{n} a_{n} t^{n} \quad \text { and } \quad A(t ; \mathbf{x})=\sum_{n, \mathbf{i}} a_{n ; \mathbf{i}} t^{n} \mathbf{x}^{\mathbf{i}} ?
$$

The answers to these questions are not simple, and heavily depend on the various parameters. The aim of this text is to provide a survey of recent results, notably classification results and closed form expressions, obtained using Computer Algebra.

\subsection{Why count walks in cones?}

Lattice paths are fundamental objects in combinatorics. They have been studied at least since the second half of the 19th century, in connection with the so-called ballot problem [64]. Various approaches have progressively been involved in the study of lattice walks, separately or in interaction. These methods arise from various fields of classical mathematics (algebra, combinatorics, complex analysis, probability theory), and more recently from computer science. There are several reasons for the ubiquity of lattice walks, but the main one is that they encode several important classes of mathematical objects, in discrete mathematics (permutations, trees, words, urns), in statistical physics (magnetism, polymers), in probability theory (branching processes, games of chance) and in operations research (birth-death processes, queueing theory). Therefore, many questions from all these various fields can be reduced to solving lattice path problems. Several books are entirely devoted to lattice paths and their applications (e.g., [35, 36, 59, 60]). For more details, the reader is referred to the introduction of [3].

\subsection{Blending experimental mathematics and computer algebra}

The enumeration of lattice walks is still a topical issue, with a lot of recent activity, new and exciting results, and many open questions. Even if we only count articles published since 2000, there are hundreds of references in this area; significant progress has been obtained by many authors, see [9] and [48] for an extensive bibliography. The dominating viewpoint in these works is to develop uniform approaches, rather than ad-hoc solutions to specific questions. My personal contribution is to combine an experimental mathematics approach, as promoted in the beautiful and inspiring books by Borwein and collaborators [2, 8], with modern tools from the computer algebra arsenal $[10,41]$, in order to conjecture and prove enumerative and asymptotic results for lattice paths.

\subsection{Classification of power series}

Before stating the main results, let us recall a few definitions on (univariate and multivariate) power series.

Definition 1.2. A series $S(t)=\sum_{n=0}^{\infty} s_{n} t^{n} \in \mathbb{Q}[[t]]$ is called:

- algebraic if it is a root of a non-trivial polynomial $P \in \mathbb{Q}[t, T]$, i.e., $P(t, S(t))=0$; transcendental if it is not algebraic;

- D-finite (differentially finite, or holonomic) if it satisfies a nontrivial linear differential equation with coefficients in $Q[t]$;

- hypergeometric if the sequence $\left(s_{n}\right)_{n}$ satisfies a non-trivial linear homogeneous recurrence of order 1 with coefficients in $Q[n]$.

An important class of hypergeometric series is that of Gauss hypergeometric functions ${ }_{2} F_{1}$ with parameters $a, b, c \in Q, c \notin-\mathbb{N}$ :

$$
{ }_{2} F_{1}\left(\begin{array}{c|c}
a & b \\
c
\end{array} \mid t\right)=\sum_{n=0}^{\infty} \frac{(a)_{n}(b)_{n}}{(c)_{n}} \frac{t^{n}}{n !},
$$

where $(x)_{n}=x(x+1) \cdots(x+n-1)$ is the rising factorial. This class contains important elementary power series such as

$$
\ln (1-t)=-\sum_{n=1}^{\infty} \frac{t^{n}}{n}, \quad(1+t)^{\alpha}=\sum_{n=0}^{\infty}\left(\begin{array}{l}
\alpha \\
n
\end{array}\right) t^{n},(\alpha \in \mathbb{Q}),
$$

and less elementary ones, such as the complete elliptic integral

$$
{ }_{2} F_{1}\left(\begin{array}{c|c}
\frac{1}{2} & \frac{1}{2} \\
1 & t
\end{array}\right)=\frac{2}{\pi} \int_{0}^{1} \frac{d x}{\sqrt{\left(1-x^{2}\right)\left(1-t x^{2}\right)}}=\sum_{n=0}^{\infty}\left(\begin{array}{c}
2 n \\
n
\end{array}\right)^{2}\left(\frac{t}{16}\right)^{n} .
$$

The notion of ${ }_{2} F_{1}$ admits an obvious extension to the so-called generalized hypergeometric function ${ }_{p} F_{q}$, involving $p$ rising factorials in the numerator and $q$ rising factorials in the denominator.

These three important classes of power series (algebraic, D-finite, hypergeometric) are connected in the following ways. Hypergeometric series are $D$-finite: this is an immediate consequence of the fact that coefficient sequences of D-finite series are exactly P-recursive sequences [68]. Algebraic series are D-finite: this fact has been observed by Abel in 1827. Algorithms for the computation of differential equations satisfied by algebraic functions were studied from the complexity perspective by Chudnovsky and Chudnovsky [30], and more recently by Bostan et al. [12]. Finally, understanding which power series are simultaneously algebraic and hypergeometric is an old and difficult question. It was solved for ${ }_{2} F_{1}$ s by Schwarz [66] in 1873 and in general only one century later, by Beukers and Heckman [7]; the decision procedure is completely algorithmic.

Similar definitions for algebraicity and D-finiteness apply to multivariate power series. For instance, $S \in \mathbb{Q}[[x, y, t]]$ is algebraic if it is the root of a non-trivial polynomial $P \in \mathbb{Q}[x, y, t, T]$, and it is $D$-finite if the set of all partial derivatives of $S$ spans a finitedimensional vector space over $\mathbb{Q}(x, y, t)$, in other words if $S$ satisfies a system of linear PDEs with polynomial coefficients of the form

$\sum_{i} a_{i}(t, x, y) \frac{\partial^{i} S}{\partial x^{i}}=0, \sum_{i} b_{i}(t, x, y) \frac{\partial^{i} S}{\partial y^{i}}=0, \sum_{i} c_{i}(t, x, y) \frac{\partial^{i} S}{\partial t^{i}}=0$.

As in the univariate case, multivariate algebraic series are D-finite [54]. 


\subsection{Walks in the quarter plane}

Let us now turn back to the general problem stated in $\S 1.2$. The simplest possible cone is the full space $\mathscr{C}=\mathbb{R}^{d}$. In that case, the full generating function has the simplest possible structure: it is rational. Indeed, a simple reasoning shows that

$$
a_{n}=|\mathscr{S}|^{n} \text {, i.e. } A(t)=\sum_{n \geq 0} a_{n} t^{n}=\frac{1}{1-|\mathscr{S}| t},
$$

and, more precisely, $A(t ; \mathbf{x})=\sum_{n, \mathbf{i}} a_{n ; \mathbf{i}} \mathbf{x}^{\mathrm{i}} t^{n}=1 /\left(1-t \sum_{\mathbf{s} \in \mathscr{S}} \mathbf{x}^{\mathbf{s}}\right)$.

The next case by increasing order of difficulty is when the cone is a half-space, typically $\mathbb{R}^{d-1} \times \mathbb{R}_{\geq 0}$. The full generating function $A(t ; \mathbf{x})$ is generally not rational anymore, but is nevertheless still algebraic. This result is due to Bousquet-Mélou and Petkovšek, see [25, Theorem 13]. The main ingredient in the proof is the socalled kernel method. A powerful generalization of this method is presented in [23], itself a special case of Popescu's theorem on Artin approximation with nested conditions [63, Thm. 1.4].

Next comes the case of a cone obtained as the intersection of two half-spaces. Up to modifying the step set by a linear transformation, one may assume that the cone is the basic orthant $\mathscr{C}=\mathbb{R}_{\geq 0}^{d}$. Quite surprisingly at first sight, generating functions for walks constrained to evolve in an orthant need not be algebraic, and not even D-finite. The first model of walks for which the generating function was proved to be non-D-finite (by Bousquet-Mélou and Petkovšek, in [26, §3]) is the so-called knight walks model: these are walks confined to $\mathbb{N}^{2}$ that start from $p_{0}=(1,1)$ and take their steps in $\mathscr{S}=\{(2,-1),(-1,2)\}$. This surprising result was the starting point of a massive classification effort, initiated by Mishna [57], intensified in a seminal work by Bousquet-Mélou and Mishna [24], and continued by many researchers.

From now on, we focus on small-step walks (or nearest-neighbor walks) in the quarter plane. These are walks in the lattice $\mathbb{Z}^{2}$, confined to the cone $\mathscr{C}=\mathbb{R}_{+}^{2}$ (we will often say confined to $\mathbb{N}^{2}$ ), that start at $p_{0}=(0,0)$ and use steps in a model $\mathscr{S}$ which is a fixed subset of $\{\swarrow, \leftarrow, \nwarrow, \uparrow, \nearrow, \rightarrow, \searrow, \downarrow\}$. Let us denote by $f_{n ; i, j}$ the number of walks of length $n$ ending at $(i, j)$. The full counting sequence $\left(f_{n ; i, j}\right)_{n, i, j}$ admits several interesting specializations:

- $e_{n}:=f_{n ; 0,0}$, the number of walks of length $n$ returning to $(0,0)$;

- $f_{n}:=\sum_{i, j \geq 0} f_{n ; i, j}$, the number of walks with prescribed length $n$.

To these enumeration sequences one attaches various power series, namely the full generating function

$$
F_{\mathscr{S}}(t ; x, y)=\sum_{n=0}^{\infty}\left(\sum_{i, j=0}^{\infty} f_{n ; i, j} x^{i} y^{j}\right) t^{n} \in \mathbb{Q}[x, y][[t]],
$$

and its corresponding univariate specializations:

- $F_{\mathscr{S}}(t ; 0,0):=\sum_{n \geq 0} e_{n} t^{n}$, the "excursion generating function";

- $F_{\mathscr{S}}(t ; 1,1):=\sum_{n \geq 0} f_{n} t^{n}$, the "length generating function";

- $F_{\mathscr{S}}(t ; 1,0)$, resp. $F_{\mathscr{S}}(t ; 0,1)$, the generating function of walks ending on the horizontal, resp. vertical, axis ("boundary returns");

- " $F_{\mathscr{S}}(t ; 0, \infty)$ " : $=\left[x^{0}\right] F_{\mathscr{S}}(t ; x, 1 / x)$, the generating function of walks ending on the diagonal $x=y$ of $\mathbb{N}^{2}$ (“diagonal returns").

(Here, and in all the text, $\left[x^{n}\right]$ denotes coefficient extraction of $x^{n}$.)

The general questions formulated in $\S 1.2$ specialize to the quarterplane setting as follows: given the model $\mathscr{S}$, what can be said about the generating function $F_{\mathscr{S}}(t ; x, y)$, resp. about the counting

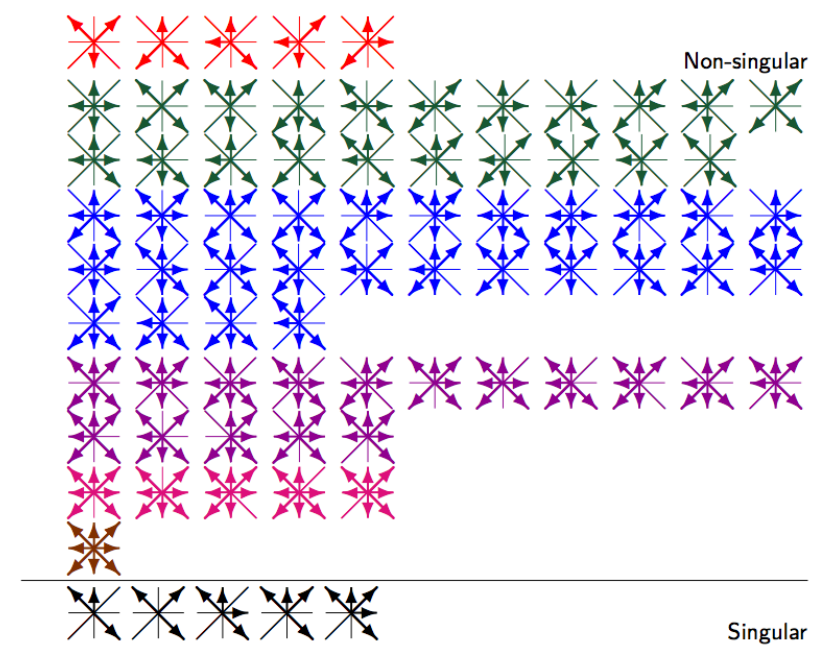

Figure 2: The 79 models of small-step walks in the quarter plane: 74 non-sigular, 5 singular.

sequence $\left(f_{n ; i, j}\right)_{i, j, n}$, and their specializations? More precise subquestions concern structures, explicit forms and asymptotics:

- Structures: is $F_{\mathscr{S}}$ algebraic? Is it D-finite?

- Explicit forms: do $F_{\mathscr{S}}(t ; x, y)$ and $\left(f_{n ; i, j}\right)_{i, j, n}$ (or some of their specializations) admit closed-form expressions?

- Asymptotics: how do $\left(e_{n}\right)_{n}$ and $\left(f_{n}\right)_{n}$ behave when $n \rightarrow \infty$ ?

\subsection{Small-step models of interest}

Among the $2^{8}$ models $\mathscr{S} \subseteq\{-1,0,1\}^{2} \backslash\{(0,0)\}$, some are trivial (e.g., if $\mathscr{S} \subseteq\{\swarrow, \leftarrow, \backslash, \searrow, \downarrow\}$, then $\left.F_{\mathscr{S}}(t ; x, y) \equiv 1\right)$, others are intrinsic to the half-plane (therefore $F_{\mathscr{S}}(t ; x, y)$ is algebraic), others come in pairs by diagonal symmetry (if $\mathscr{S}$ and $\mathscr{S}^{\prime}$ are symmetric with respect to the diagonal of $\mathbb{N}^{2}$, then $\left.F_{\mathscr{S}}(t ; x, y) \equiv F_{\mathscr{S}^{\prime}}(t ; y, x)\right)$.

After discarding these cases, Bousquet-Mélou and Mishna [24] found that there are 79 interesting distinct models of small-step walks in the quarter plane. They are represented in Fig. 2 and are grouped in two classes: 74 non-singular models (or genus-1 models) and 5 singular models (or genus-0 models). Singular models are the ones for which walks never return to the origin, that is for which the excursions generating function is trivial $F(t ; 0,0) \equiv 1$.

Among the 79 models, a few "special" ones are considered interesting enough and were sufficiently studied in order to deserve names (as "special functions" do): Tandem:

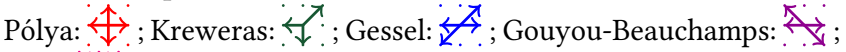
King: The objective is to classify all these 79 models according to the structural properties of their generating functions.

\subsection{Two nice models: Kreweras' and Gessel's}

An interesting model in the world of quarter-plane walks is Kreweras' model $\mathscr{S}=\{\downarrow, \leftarrow, \nearrow\}$. It is related to a version of the threecandidate ballot problem [61] and also to an applied probabilistic context [39] (double queue that arises when arriving customers simultaneously place two demands handled independently by two 
servers). In a long paper, Kreweras [49] obtained the following result, which was reproved using various methods in $[5,16,20,21,42$, $45,50,61]$. References $[16,45]$ provide two different computer-aided proofs. In what follows, we denote by $K(t ; x, y)=F_{\mathscr{S}}(t ; x, y)$ the full generating function for Kreweras walks in the quarter plane, and by $K(t ; 0,0)$ the generating function for Kreweras excursions.

Theorem 1.3 ([49]). $K(t ; 0,0)=1+2 t^{3}+16 t^{6}+\cdots$ is equal to

$$
{ }_{3} F_{2}\left(\begin{array}{ccc|}
\frac{1}{3} & \frac{2}{3} & 1 \\
\frac{3}{2} & 2
\end{array} \mid 27 t^{3}\right)=\sum_{n=0}^{\infty} \frac{4^{n}\left(\begin{array}{c}
3 n \\
n
\end{array}\right)}{(n+1)(2 n+1)} t^{3 n} .
$$

Theorem 1.3 implies, together with the results in $§ 1.5$ (e.g. [7]) that $K(t ; 0,0)$ is an algebraic power series. In fact, much more is true:

Theorem $1.4([21,39,42]) . K(t ; x, y)$ is algebraic.

The most difficult model of small-step walks in the quarter plane is Gessel's model $\mathscr{S}=\{\nearrow, \swarrow, \leftarrow, \rightarrow\}$. In 2001, Ira Gessel formulated two conjectures equivalent to the following statements:

Conjecture 1. The generating function $G(t ; 0,0)=1+2 t^{2}+$ $11 t^{4}+85 t^{6}+782 t^{7}+\cdots$ of Gessel excursions is equal to

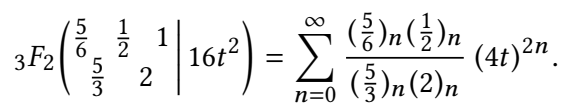

Conjecture 2. The generating function $G(t ; x, y)$ is not $D$-finite.

Here, $G(t ; x, y)=F_{\mathscr{S}}(t ; x, y)$ for $\mathscr{S}=\{\nearrow, \swarrow, \leftarrow, \rightarrow\}$ denotes the full generating function for Gessel walks in the quarter plane, and $G(t ; 0,0)$ denotes the generating function for Gessel excursions.

\subsection{Algebraic translation: functional equations}

Gessel's problem admits the following purely algebraic reformulation. If $G(t ; x, y) \in \mathbb{Q}[x, y][[t]]$ denotes the full generating function for Gessel walks in the quarter plane, then a simple inclusionexclusion reasoning represented pictorially in Fig. 3 implies that $G(t ; x, y)$ satisfies a functional equation called the kernel equation

$$
\begin{aligned}
G(t ; x, y)= & +t\left(x y+x+\frac{1}{x y}+\frac{1}{x}\right) G(t ; x, y) \\
& -t\left(\frac{1}{x}+\frac{1}{x} \frac{1}{y}\right) G(t ; 0, y)-t \frac{1}{x y}(G(t ; x, 0)-G(t ; 0,0))
\end{aligned}
$$

Moreover, $G(t ; x, y)$ is completely characterized by the functional equation (4): it is its unique solution in $\mathbb{Q}[x, y][[t]]$, and even in the ring $\mathbb{Q}[[x, y, t]]$. Therefore, the task is simply to solve equation (4).

To any of the 79 models introduced in $\S 1.7$ is attached a very similar functional equation. Again, this equation merely reflects a step-by-step construction of quarter-plane walks, and is based on the most elementary decomposition: a walk is either the empty walk, or a shorter walk followed by a permissible step. This observation is naturally translated into a generating function equation using the inventory $\chi \mathscr{S}(x, y):=\sum_{(i, j) \in \mathscr{S}} x^{i} y^{j}$, and the kernel $\mathfrak{\Omega}_{\mathscr{S}}(t ; x, y):=$ $x y(1-t \chi \mathscr{S}(x, y))$. The decomposition is translated into the kernel equation (we omit the subscript $\mathscr{S}$ ):

$$
\begin{aligned}
\Re(t ; x, y) F(t ; x, y)= & x y+\Re(t ; x, 0) F(t ; x, 0) \\
& +\Re(t ; 0, y) F(t ; 0, y)-\Re(t ; 0,0) F(t ; 0,0) .
\end{aligned}
$$

Observe that the last term of the right-hand side occurs only if the step $\swarrow$ belongs to the model $\mathscr{S}$.
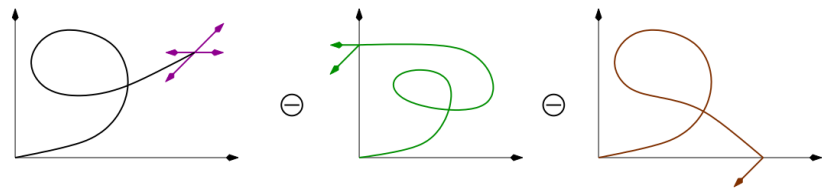

Figure 3: Functional equation for Gessel walks, pictorially.

\section{MAIN RESULTS}

In $\S 1.9$ we saw that classifying lattice walks in the quarter plane amounts to solving 79 equations of the form (5). In this section we describe several classes of results that have been obtained, using computer algebra tools, on the solutions of these 79 equations.

\subsection{Algebraicity of Gessel walks}

After a first attempt in [45], Gessel's first conjecture was finally solved in 2009 by Kauers, Koutschan and Zeilberger in [44] using an extension of the guess-and-prove approach described in [45].

Theorem 2.1 ([44]). $G(t ; 0,0)={ }_{3} F_{2}\left(\begin{array}{ccc|c}5 / 6 & 1 / 2 & 1 / 2 \\ 5 / 3 & 2 & 16 t^{2}\end{array}\right)$.

This result implies in particular that $G(t ; 0,0)$ is D-finite, but has no immediate implications concerning the D-finiteness of $G(t ; x, y)$. It came as a total surprise when Bostan and Kauers [16] proved that Gessel's second conjecture was false.

THEOREM 2.2 ([16]). The generating function $G(t ; x, y)$ for Gessel walks is algebraic.

Prior to this result, even the algebraicity of $G(t ; 0,0)$ had been overlooked, even though the classical results recalled in $§ 1.5$ apply. The original discovery and proof of Theorem 2.2 was computerdriven, and used a guess-and-prove approach, based on HermitePadé approximants [16]. Note that as a byproduct of this proof, an estimate on the size of the minimal polynomial of $G(t ; x, y)$ has been given: it has more than $10^{11}$ terms when written in dense (expanded) form, for a total size of $\approx 30 \mathrm{~Gb}(!)$. The guess-and-prove method is a 3-step process:

(S1) compute $G(t ; x, y)$ to precision $t^{1200}(\approx 1.5$ billion coeffs!);

(S2) conjecture polynomial equations for $G(x, 0 ; t)$ and $G(0, y ; t)$ (degree 24 each, coeffs. of degree $(46,56)$, with 80 -bit digits coeffs.), relying on the Beckermann-Labahn algorithm [4];

(S3) conclude the proof by computing multivariate resultants of (very big) polynomials (30 pages each), using fast algorithms for manipulating algebraic series [14].

Several human proofs of Theorems 2.1 and 2.2 have been discovered since the publication of [16]: the first one used complex analysis [17], the second one was purely algebraic [22], the third one is both combinatorial and analytic [28], while the more recent one is probably the most elementary [6].

\subsection{Explicit form for $G(t ; x, y)$}

An interesting refinement of Theorem 2.2 is the next result, which contains a closed-formula for the generating function $G(t ; x, y)$. 
TheOREM $2.3([16])$. Let $V=1+4 t^{2}+\cdots$ be the root in $Q[[t]]$ of $(V-1)(1+3 / V)^{3}=(16 t)^{2}$, let $U=1+2 t^{2}+16 t^{4}+2 x t^{5}+\cdots$ be the root in $Q[x][[t]]$ of

$$
\begin{aligned}
& x(V-1)(V+1) U^{3}-2 V(3 x+5 x V-8 V t) U^{2} \\
& -x V\left(V^{2}-24 V-9\right) U+2 V^{2}(x V-9 x-8 V t)=0,
\end{aligned}
$$

and let $W=t^{2}+(y+8) t^{4}+2\left(y^{2}+8 y+41\right) t^{6}+\cdots$ be the root in $Q[y][[t]]$ of $y(1-V) W^{3}+y(V+3) W^{2}-(V+3) W+V-1=0$.

Then $G(t ; x, y)$ is equal to

$$
\frac{\frac{64(U(V+1)-2 V) V^{3 / 2}}{x\left(U^{2}-V\left(U^{2}-8 U+9-V\right)\right)^{2}}-\frac{y(W-1)^{4}(1-W y) V^{-3 / 2}}{t(y+1)(1-W)\left(W^{2} y+1\right)^{2}}}{\left(1+y+x^{2} y+x^{2} y^{2}\right) t-x y}-\frac{1}{t x(y+1)} .
$$

Again, the original discovery and proof of this result was computerdriven, based on effective Galois theory. During the proof, a few other remarkable facts have been noticed, namely that $G(t ; x, y)$ can be expressed using nested radicals; for instance the length generating function $G(t ; 1,1)=1+2 t+7 t^{2}+21 t^{3}+78 t^{4}+\cdots$ reads

$$
G(t ; 1,1)=-\frac{1}{2 t}+\frac{\sqrt{3}}{6 t} \sqrt{H(t)+\sqrt{\frac{16 t(2 t+3)+2}{(1-4 t)^{2} H(t)}-H(t)^{2}+3}},
$$

where $H(t)=\sqrt{1+4 t^{1 / 3}(1+4 t)^{2 / 3} /(1-4 t)^{4 / 3}}$. The proof of Theorem 2.3 uses the minimal polynomials for $G(t ; x, 0)$ and $G(t ; 0, y)$ that were guessed and proved during the algebraicity proof. A striking feature of Theorem 2.3 is the relative simplicity of the closed-form expression, especially when compared to the size of the minimal polynomial of $G(t ; x, y)$. Similarly to Theorem 2.2 , the result in Theorem 2.3 admits several recent human proofs $[6,17,22]$.

\subsection{Models with D-finite generating functions}

The computer-driven approach that allowed Bostan and Kauers [16] to discover and prove the properties of the puzzling generating function for Gessel walks was used as early as 2008 by the same authors to provide a (conjecturally) exhaustive list of models having (conjecturally) D-finite and algebraic generating functions [15]. That resulted in an experimental classification synthesized in Fig. 4, which displays 23 models of walks in the quarter plane for which the length generating function $F(t ; 1,1)$ was conjectured to be $\mathrm{D}$-finite. The computerized discovery used again (differential) guessing, based on Hermite-Padé approximation. Note that the guess-and-prove method used to prove Theorem 2.2 is robust enough to also prove that all models in Fig. 4 are indeed D-finite (with resultant computations in (S3) replaced by differential closure algorithms). The labels used in column "OEIS" are taken from Sloane's On-Line Encyclopedia of Integer Sequences [67]. The "alg?" column refers to the algebraicity or transcendence of $F(t ; 1,1)$ : cases marked "Y" were conjectured algebraic (using algebraic Hermite-Padé approximation), the other cases were conjectured transcendental. For cases $1-22$, these results on D-finiteness, resp. algebraicity, were confirmed by human proofs obtained almost simultaneously with [15] by Bousquet-Mélou and Mishna [24], using a uniform approach. We discussed the difficult case 23 (Gessel's model) in §2.1 and §2.2. Concerning the conjectural transcendence results, the first unified proof was given in [13] and it is computer-driven; this will be discussed in $\S 2.6$. The reference [13] also contains the first proof,

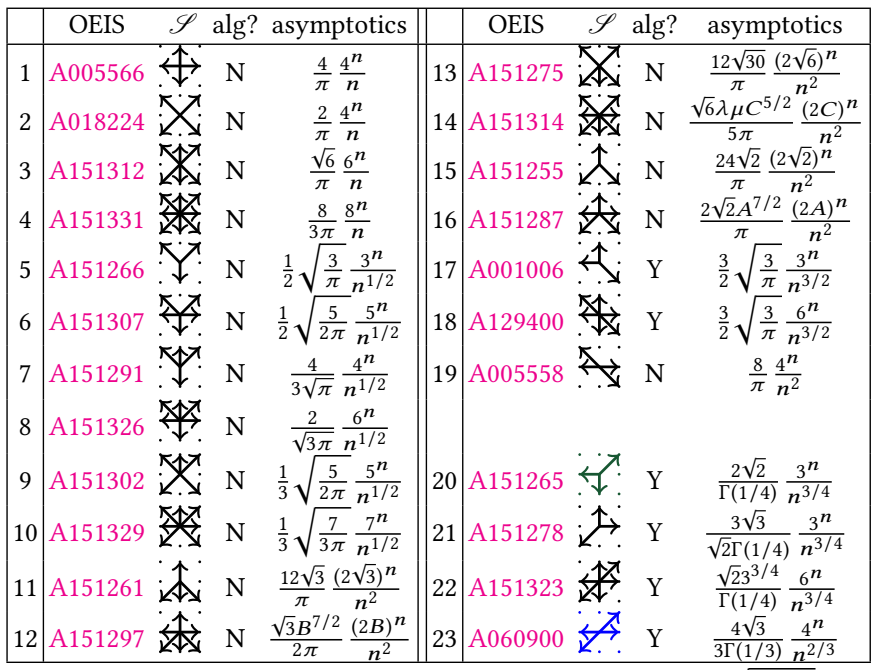

$A=1+\sqrt{2}, B=1+\sqrt{3}, C=1+\sqrt{6}, \lambda=7+3 \sqrt{6}, \mu=\sqrt{\frac{4 \sqrt{6}-1}{19}}$

Figure 4: Models with D-finite length generating function: algebraicity of $F_{\mathscr{S}}(t ; 1,1)$, asymptotics of $f_{n}=\left[t^{n}\right] F_{\mathscr{S}}(t ; 1,1)$. For models 11, 13 and 15, estimates only hold for even $n$; for odd $n$, the constants change into $\frac{18}{\pi}, \frac{144}{\sqrt{5}}$ and $\frac{32}{\pi}$ [56].

again computer-driven, that the (differential / recurrence / algebraic) equations conjectured in [15] are indeed correct.

In addition, Bostan and Kauers demonstrated that computer algebra tools are also able to produce conjectural expressions for the asymptotics of $f_{n}=\left[t^{n}\right] F(t ; 1,1)$. Their results are displayed in column "asymptotics" of Fig. 4 and have been obtained using a combination of algorithmic tools, including Hermite-Padé approximation, constant recognition algorithms built on integer relation detection algorithms like LLL [53] and PSLQ [37], and convergence acceleration techniques [27]. These results have been confirmed a few years later by human proofs by Melczer and Wilson [56], using the theory of analytic combinatorics in several variables [62] that extends the univariate theory from [38].

\subsection{The group of a model}

In order to formulate more results on the classification of lattice walks in the quarter plane, we need to introduce an important concept, the group of a small-step walk model $\mathscr{S}$. Let us decompose the inventory $\chi_{\mathscr{S}}$ of $\mathscr{S}$ along powers of $x$, resp. of $y$, as follows:

$$
\chi_{\mathscr{S}}(x, y)=\sum_{(i, j) \in \mathscr{S}} x^{i} y^{j}=\sum_{i=-1}^{1} B_{i}(y) x^{i}=\sum_{j=-1}^{1} A_{j}(x) y^{j} .
$$

The basic, yet fundamental, observation is that $\chi \mathscr{S}(x, y)$ is left invariant under two rational transformations

$$
\psi(x, y)=\left(x, \frac{A_{-1}(x)}{A_{+1}(x)} \frac{1}{y}\right), \quad \phi(x, y)=\left(\frac{B_{-1}(y)}{B_{+1}(y)} \frac{1}{x}, y\right),
$$

and thus under any element of the group $\mathcal{G S}_{\mathscr{S}}:=\langle\psi, \phi\rangle$ of birational transformations generated by $\psi$ and $\phi$. When it is finite, $\mathcal{G}_{\mathscr{S}}$ is isomorphic to a dihedral group, since $\psi$ and $\phi$ are involutions. Mishna [57] showed that for the 23 models in Fig. 4, the group is 
finite, of cardinality either 4 (for models $1-16$ with an axial symmetry), 6 (for the models 17, 18, 20,21, 22, with a diagonal or an anti-diagonal symmetry), or 8 (for the remaining models 19 and 23). Bousquet-Mélou and Mishna [24] proved in addition that for all the other 56 models, the group is infinite.

At this point, we know that the finiteness of the group for some model implies the D-finiteness of the generating function for that model. One important remaining question is: is the converse true? Another important question is: in the D-finite cases, are there any closed-form expressions for the generating functions? The next two subsections will bring answers and completely clarify the situation.

\subsection{Explicit expressions}

Models $20-23$ in Fig. 4 admit full generating functions that are algebraic. Moreover, closed formulas exist for them. For the three models 20-22 related to the Kreweras model, such formulas are displayed in [24, §6]. The most difficult case among these four is model 23 (Gessel's), for which Theorem 2.3 provides a closed-form expression. We now focus on models 1-19. The natural question is whether closed-form expressions also exist in these cases. This question has recently received a positive answer using computer algebra tools in [13]: $F_{\mathscr{S}}$ is uniformly expressible using iterated integrals of hypergeometric ${ }_{2} F_{1}$ expressions. More precisely, the following structure result, already conjectured in [15, §3.2], holds.

Theorem 2.4 ([13]). Let $\mathscr{S}$ be one of the models 1-19 in Fig. 4. Then $F_{\mathscr{S}}(t ; x, y)$ is expressible as a finite sum of iterated integrals of products of algebraic functions in $x, y, t$ and of expressions of the

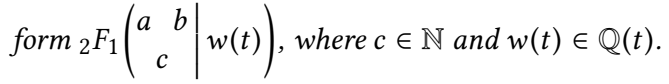

Once again, the discovery and proof of this result are computerdriven; no human proof is available yet. The proof is based, among other tools, on creative telescoping, an efficient algorithmic technique for the symbolic integration of multivariate functions with parameters. This powerful computer algebra tool was introduced in the early 1990s by Zeilberger in the "hyper" setting [70, 71], vastly generalized by Chyzak in the 2000s to the framework of holonomic functions [31], and further enhanced by Koutschan in the 2010s [46]. Since its birth, 30 years ago, the methodology of creative telescoping gained more and more popularity. To date, there are no less than four generations of creative telescoping algorithms; examples of $4 \mathrm{G}$ integration algorithms are in [18] and [11]. For more details, we refer the reader to the surveys [29, 32, 47].

Using the kernel method, Bousquet-Mélou and Mishna showed in $\left[24\right.$, Prop. 8] that the generating function $F_{\mathscr{S}}(t ; x, y)$ for models $1-$ 19 can be written in terms of the positive part of a rational function,

$$
F_{\mathscr{S}}(t ; x, y)=\frac{1}{x y}\left[x^{>}\right]\left[y^{>}\right] \frac{N(x, y)}{1-t S(x, y)},
$$

where $N(x, y)$ and $S(x, y)$ are certain Laurent polynomials in $y$ with coefficients that are rational functions in $x$. By a result of Lipshitz [54, Thm. 2.7], Equation (6) already implies the D-finiteness of $F_{\mathscr{S}}(t ; x, y)$. To obtain explicit differential equation for $F_{\mathscr{S}}(t ; x, y)$ and its specializations, the key is creative telescoping. Once a linear differential equation is obtained, the last steps in the proof of Theorem 2.4 rely on factoring differential operators [69] and on solving second-order differential equations in terms of ${ }_{2} F_{1} \mathrm{~s}[43,51]$.
The expressions of the generating functions $F(t ; 0,0), F(t ; 0,1)$, $F(t ; 1,0), F(t ; 1,1), F(t ; x, 0), F(t ; 0, y)$ and $F(t ; x, y)$ are too large to be displayed here, and are available on-line. It turns out that the involved hypergeometric functions have a very particular form: they are intimately related to elliptic integrals such as (2). For instance, for King walks (model 4), the length generating function is

$$
F_{\text {速 }}(t ; 1,1)=\frac{1}{t} \int_{0}^{t} \frac{1}{(1+4 x)^{3}} \cdot{ }_{2} F_{1}\left({ }^{\frac{3}{2}}{ }^{\frac{3}{2}} \mid \frac{16 x(1+x)}{(1+4 x)^{2}}\right) d x \text {. }
$$

No human proof is currently available for the structure Theorem 2.4, and even less for the explicit expressions implied by it. However, the book [36] probably contains the premises of the key explanation: the kernel for models 1-19 is an elliptic curve, therefore the solution of the kernel equation (5) should be related to elliptic integrals.

\subsection{Transcendence results}

As previously mentioned, models $20-23$ in Fig. 4 admit full generating functions that are algebraic. What about the full generating function $F_{\mathscr{S}}(t ; x, y)$, and its combinatorially meaningful specializations $F_{\mathscr{S}}(t ; 0,0), F_{\mathscr{S}}(t ; 1,0), F_{\mathscr{S}}(t ; 0,1)$ and $F_{\mathscr{S}}(t ; 1,1)$ for the models $1-19$ ? Computer algebra is able to answer this question.

Theorem 2.5 ([13]). Let $\mathscr{S}$ be one of the models 1-19 in Fig. 4. Then for any $(\alpha, \beta) \in\{(0,0),(1,0),(0,1),(1,1)\}$, the power series $F_{\mathscr{S}}(t ; \alpha, \beta)$ is transcendental, except in the following four cases:

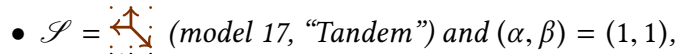

- $\mathscr{S}=$ (model 18) and $(\alpha, \beta) \in\{(1,0),(0,1),(1,1)\}$.

As a consequence, $F_{\mathscr{S}}(t ; x, y), F_{\mathscr{S}}(t ; x, 0)$, and $F_{\mathscr{S}}(t ; 0, y)$ are transcendental for all the 19 models. Additionally, the generating functions of the four algebraic cases are equal to:

$$
\begin{aligned}
& \text { - } F(t ; 1,1)=\frac{1}{2 t^{2}}(1-t-\sqrt{(1+t)(1-3 t)}), \\
& \text { - }(t ; 1,1)=F(2 t ; 1,1), \\
& \text { - } F_{i}(t ; 1,0)=F(t ; 0,1)=\frac{(1-6 t)^{3 / 2}(1+2 t)^{1 / 2}-4 t^{2}+8 t-1}{32 t^{3}} \text {. }
\end{aligned}
$$

Again, the proof of Theorem 2.5 is computer-driven and heavily relies on the use of several modern computer algebra algorithms. Transcendence proofs had been considered in some isolated cases only. The computer-aided proof of Theorem 2.5 was the first complete and unified transcendence proof applying to all 19 models.

\subsection{Non-D-finiteness results}

The last question in view of the complete classification of small step walks in the quarter plane concerns the 56 models with an infinite group. Among them, 5 models are singular; for them, a variant of the kernel method, called the iterated kernel method was used by Mishna and Rechnitzer [58] (for two models) and by Melczer and Mishna [55] (for all five models), who showed that the length generating function $F(t ; 1,1)$, and thus also the full generating function $F(t ; x, y)$, are non-D-finite. The remaining question concerns the 51 non-singular models with an infinite group: is the full generating function again non-D-finite?

Computer algebra is able to help prove the following result.

Theorem 2.6 ([19]). Let $\mathscr{S} \subseteq\{0, \pm 1\}^{2}$ be any of the 51 nonsingular step sets in $\mathbb{N}^{2}$ with infinite group $\mathcal{G}_{\mathscr{S}}$. Then the generating 


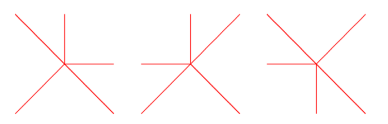

Figure 5: Rotations of a scarecrow: models with zero drift that have a non-D-finite generating function.

function $F_{\mathscr{S}}(t ; 0,0)$ of $\mathscr{S}$-excursions is not $D$-finite. Equivalently, the excursion sequence $\left(e_{n}\right)_{n \geq 0}$ is not P-recursive.

In particular, the full generating function $F_{\mathscr{S}}(t ; x, y)$ is not Dfinite in the 51 cases, since D-finiteness is preserved by specialization [54]. This corollary had been already obtained by Kurkova and Raschel [52], but the approach in [19] is simpler, and at the same time it delivers a more accurate information. This new proof combines asymptotic information about the coefficients $e_{n}$ of $F_{\mathscr{S}}(t ; 0,0)$, and arithmetic information about the constrained behavior of the asymptotics of $e_{n}$ when $F_{\mathscr{S}}(t ; 0,0)$ is D-finite. More precisely, [19] first makes explicit consequences of the general results by Denisov and Wachtel [34] in the case of walks in the quarter plane. This analysis implies that, when $n$ tends to infinity, $e_{n}$ behaves like $\kappa \cdot \rho^{n} \cdot n^{\alpha}$, where $\kappa=\kappa(\mathscr{S})>0$ is a real number, $\rho=\rho(\mathscr{S})$ is an algebraic number, and $\alpha=\alpha(\mathscr{S})$ is a real number such that $c=-\cos \left(\frac{\pi}{1+\alpha}\right)$ is an algebraic number. More explicitly, $\rho:=\chi\left(x_{0}, y_{0}\right)$ and

$$
\alpha:=-1-\frac{\pi}{\arccos (-c)} \quad \text { with } \quad c:=\frac{\frac{\partial^{2} \chi}{\partial x \partial y}}{\sqrt{\frac{\partial^{2} \chi}{\partial x^{2}} \cdot \frac{\partial^{2} \chi}{\partial y^{2}}}}\left(x_{0}, y_{0}\right),
$$

where $\left(x_{0}, y_{0}\right)$ is the unique solution in $\mathbb{R}_{>0}^{2}$ of $\frac{\partial \chi}{\partial x}=\frac{\partial \chi}{\partial y}=0$.

Starting from the step set $\mathscr{S}$, real approximations for $\rho, \alpha$ and $c$ can be determined to arbitrary precision. Moreover, exact minimal polynomials of $\rho$ and $c$ can be determined algorithmically, using tools from elimination theory, namely Gröbner bases [33]. A classical result in the arithmetic theory of linear differential equations [40] about the possible asymptotic behavior of an integervalued, exponentially bounded D-finite sequence, states that if such a sequence grows like $\kappa \cdot \rho^{n} \cdot n^{\alpha}$, then $\alpha$ is necessarily a rational number. For the 51 cases of nonsingular walks with infinite group, [19] proves that the constant $\alpha=\alpha(\mathscr{S})$ is not a rational number; the proof ultimately amounts to checking that some explicit polynomials in $\mathbb{Q}[t]$, obtained by algebraic elimination, are not cyclotomic; this is a task that can be performed algorithmically [1].

Example 2.7. Consider the three scarecrows models depicted in Fig. 5. For the first and the third, the approach sketched above shows that the excursions sequence $\left(e_{n}\right)_{n}=(1,0,0,2,4,8,28,108, \ldots)$ is asymptotically $\sim \kappa \cdot 5^{n} \cdot n^{\alpha}$, for $\alpha=-1-\frac{\pi}{\arccos (1 / 4)}=-3.383396 \ldots$ The irrationality of $\alpha$ prevents $F_{\mathscr{S}}(t ; 0,0)$ from being D-finite.

\subsection{Summary: classification of $2 \mathrm{D}$ walks}

Definition 2.8. The orbit sum of a quarter-plane model $\mathscr{S}$ with finite group $\mathcal{G S S}_{\mathscr{S}}$ is the following polynomial in $\mathbb{Q}\left[x, x^{-1}, y, y^{-1}\right]$ :

$$
\operatorname{OS}_{\mathscr{S}}:=\sum_{g \in \mathcal{G}_{\mathscr{S}}}(-1)^{g} g(x) g(y)
$$

where for $g \in G_{\mathscr{S}}$ we write $(-1)^{g}$ for the sign of $g$, i.e., 1 if $g$ is the product of an even number of generators $\phi$ and $\psi$, and else -1 .

A simple computation shows that for exactly the four models $20-23$, the orbit sum is zero. E.g., for the Kreweras model:

$$
\text { OS } \underset{7}{ }=x \cdot y-\frac{1}{x y} \cdot y+\frac{1}{x y} \cdot x-y \cdot x+y \cdot \frac{1}{x y}-x \cdot \frac{1}{x y}=0 .
$$

We now state the main result of this text, obtained by combining all previous results. It provides a complete characterization of the non-singular small-step sets with D-finite full generating function. The drift of a model $\mathscr{S}$ is defined as the sum of the vectors in $\mathscr{S}$.

Theorem 2.9. Let $\mathscr{S} \subseteq\{0, \pm 1\}^{2}$ be any of the 74 nonsingular quarter-plane models in Fig. 2. The following assertions are equivalent:

(1) the full generating function $F_{\mathscr{S}}(t ; x, y)$ is D-finite;

(2) the excursions generating function $F_{\mathscr{S}}(t ; 0,0)$ is $D$-finite;

(3) the sequence $\left[t^{2 n}\right] F_{\mathscr{S}}(t ; 0,0)$ is $\sim K \cdot \rho^{n} \cdot n^{\alpha}$, with $\alpha \in \mathbb{Q}$;

(4) the group $\mathcal{G}_{\mathscr{S}}$ is finite;

(5) $\mathscr{S}$ has either an axial symmetry, or zero drift and $|\mathscr{S}| \neq 5$.

Moreover, under (1)-(5), $\left|\mathcal{G S}_{\mathscr{S}}\right|$ is equal to $2 \cdot \min \left\{\ell \in \mathbb{N}_{>0} \mid \frac{\ell}{\alpha+1} \in \mathbb{Z}\right\}$.

Still under (1)-(5), $F_{\mathscr{S}}(t ; x, y)$ is algebraic if and only if $O S_{\mathscr{S}}=0$. In this case, $F_{\mathscr{S}}(t ; x, y)$ is expressible using nested radicals. Otherwise, $F_{\mathscr{S}}(t ; x, y)$ is expressible using iterated integrals of ${ }_{2} F_{1}$ expressions.

Acknowledgements. I am grateful to Frédéric Chyzak, Bogdan Marin, Marc Mezzarobba and Sergey Yurkevich for their careful reading and for their valuable remarks. This work was supported by DeRerumNatura ANR-19-CE40-0018.

\section{BIOGRAPHY}

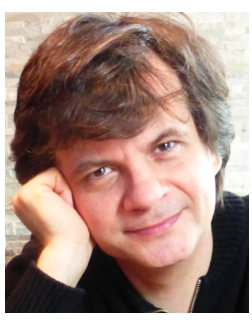

Alin Bostan is a computer scientist and mathematician working at Inria in France. His main research field is symbolic computation, notably the design of efficient algebraic algorithms. He blends an experimental mathematics approach with fast computer algebra algorithms in order to solve problems from enumerative combinatorics, statistical physics, and number theory.

\section{REFERENCES}

[1] A. Arnold and M. Monagan. Calculating cyclotomic polynomials. Math. Comp., 80(276):2359-2379, 2011.

[2] D. H. Bailey, J. M. Borwein, N. J. Calkin, R. Girgensohn, D. R. Luke, and V. H. Moll. Experimental mathematics in action. A K Peters, Ltd., Wellesley, MA, 2007.

[3] C. Banderier and P. Flajolet. Basic analytic combinatorics of directed lattice paths. Theoret. Comput. Sci., 281(1-2):37-80, 2002.

[4] B. Beckermann and G. Labahn. A uniform approach for the fast computation of matrix-type Padé approximants. SIAM J. Matrix Anal. Appl., 15(3):804-823, 1994.

[5] O. Bernardi. Bijective counting of Kreweras walks and loopless triangulations. J. Combin. Theory Ser. A, 114(5):931-956, 2007.

[6] O. Bernardi, M. Bousquet-Mélou, and K. Raschel. Counting quadrant walks via Tutte's invariant method. To appear in Combinatorial Theory.

[7] F. Beukers and G. Heckman. Monodromy for the hypergeometric function ${ }_{n} F_{n-1}$. Invent. Math., 95(2):325-354, 1989.

[8] J. Borwein and K. Devlin. The computer as crucible. A K Peters, Ltd., Wellesley, MA, 2009. An introduction to experimental mathematics.

[9] A. Bostan. Computer Algebra for Lattice Path Combinatorics. HDR (accreditation to supervise research), Université Paris 13, 2017. 79 pages. 
[10] A. Bostan, F. Chyzak, M. Giusti, R. Lebreton, G. Lecerf, B. Salvy, and E. Schost Algorithmes Efficaces en Calcul Formel. Palaiseau, Sept. 2017. 686 pages. Book printed by CreateSpace. Also available in electronic version.

[11] A. Bostan, F. Chyzak, P. Lairez, and B. Salvy. Generalized Hermite reduction, creative telescoping and definite integration of D-finite functions. In Proc. ISSAC'18, pages 95-102. ACM, 2018.

[12] A. Bostan, F. Chyzak, G. Lecerf, B. Salvy, and E. Schost. Differential equations for algebraic functions. In Proc. ISSAC'07, pages 25-32. ACM, 2007.

[13] A. Bostan, F. Chyzak, M. van Hoeij, M. Kauers, and L. Pech. Hypergeometric expressions for generating functions of walks with small steps in the quarter plane. European J. Combin., 61:242-275, 2017.

[14] A. Bostan, P. Flajolet, B. Salvy, and É. Schost. Fast computation of special resultants. Journal of Symbolic Computation, 41(1):1-29, January 2006.

[15] A. Bostan and M. Kauers. Automatic classification of restricted lattice walks. In DMTCS Proc. FPSAC'09, pages 203-217, 2009.

[16] A. Bostan and M. Kauers. The complete generating function for Gessel walks is algebraic. Proc. the American Mathematical Society, 138(9):3063-3078, 2010. With an Appendix by M. van Hoeij.

[17] A. Bostan, I. Kurkova, and K. Raschel. A human proof of Gessel's lattice path conjecture. Trans. Amer. Math. Soc., 369(2):1365-1393, 2017.

[18] A. Bostan, P. Lairez, and B. Salvy. Creative telescoping for rational functions using the Griffiths-Dwork method. In Proc. ISSAC'13, pages 93-100. ACM, 2013.

[19] A. Bostan, K. Raschel, and B. Salvy. Non-D-finite excursions in the quarter plane J. Combin. Theory Ser. A, 121:45-63, 2014.

[20] M. Bousquet-Mélou. Counting walks in the quarter plane. In Mathematics and computer science, II (Versailles, 2002), Trends Math., pages 49-67. Birkhäuser, Basel, 2002.

[21] M. Bousquet-Mélou. Walks in the quarter plane: Kreweras' algebraic model. Ann. Appl. Probab., 15(2):1451-1491, 2005.

[22] M. Bousquet-Mélou. Square lattice walks avoiding a quadrant. J. Combin. Theory Ser. A, 144:37-79, 2016.

[23] M. Bousquet-Mélou and A. Jehanne. Polynomial equations with one catalytic variable, algebraic series and map enumeration. J. Combin. Theory Ser. B, 96(5):623$672,2006$.

[24] M. Bousquet-Mélou and M. Mishna. Walks with small steps in the quarter plane. In Algorithmic probability and combinatorics, volume 520 of Contemp. Math. pages 1-39. Amer. Math. Soc., Providence, RI, 2010.

[25] M. Bousquet-Mélou and M. Petkovšek. Linear recurrences with constant coefficients: the multivariate case. Discrete Math., 225(1-3):51-75, 2000. Formal power series and algebraic combinatorics (Toronto, ON, 1998).

[26] M. Bousquet-Mélou and M. Petkovšek. Walks confined in a quadrant are not always D-finite. Theoret. Comput. Sci., 307(2):257-276, 2003.

[27] C. Brezinski. Convergence acceleration during the 20th century. J. Comput. Appl Math., 122(1-2):1-21, 2000. Numerical analysis 2000, Vol. II: Interpolation and extrapolation.

[28] T. Budd. Winding of simple walks on the square lattice. J. Combin. Theory Ser. A, 172:105191, 59, 2020

[29] S. Chen and M. Kauers. Some open problems related to creative telescoping. J. Syst. Sci. Complex., 30(1):154-172, 2017.

[30] D. V. Chudnovsky and G. V. Chudnovsky. On expansion of algebraic functions in power and Puiseux series. I. J. Complexity, 2(4):271-294, 1986.

[31] F. Chyzak. An extension of Zeilberger's fast algorithm to general holonomic functions. Discrete Math., 217(1-3):115-134, 2000.

[32] F. Chyzak. The ABC of Creative Telescoping - Algorithms, Bounds, Complexity. HDR (accreditation to supervise research), University Paris-Sud, 2014. 63 pages

[33] D. Cox, J. Little, and D. O'Shea. Using algebraic geometry, volume 185 of Graduate Texts in Mathematics. Springer-Verlag, New York, 1998.

[34] D. Denisov and V. Wachtel. Random walks in cones. Ann. Probab., 43(3):992-1044, 2015

[35] P. G. Doyle and J. L. Snell. Random walks and electric networks, volume 22 of Carus Mathematical Monographs. Mathematical Association of America, 1984.

[36] G. Fayolle, R. Iasnogorodski, and V. Malyshev. Random walks in the quarter plane, volume 40 of Probability Theory and Stochastic Modelling. Springer, second edition, 2017. Algebraic methods, boundary value problems, applications to queueing systems and analytic combinatorics.

[37] H. R. P. Ferguson, D. H. Bailey, and S. Arno. Analysis of PSLQ, an integer relation finding algorithm. Math. Comp., 68(225):351-369, 1999.

[38] P. Flajolet and R. Sedgewick. Analytic combinatorics. Cambridge University Press, Cambridge, 2009.

[39] L. Flatto and S. Hahn. Two parallel queues created by arrivals with two demands I. SIAM J. Appl. Math., 44(5):1041-1053, 1984.

[40] S. Garoufalidis. $G$-functions and multisum versus holonomic sequences. Adv. Math., 220(6):1945-1955, 2009.

[41] J. von zur Gathen and J. Gerhard. Modern computer algebra. Cambridge University Press, Cambridge, third edition, 2013.

[42] I. M. Gessel. A probabilistic method for lattice path enumeration. J. Statist. Plann. Inference, 14(1):49-58, 1986.
[43] E. Imamoglu and M. van Hoeij. Computing hypergeometric solutions of second order linear differential equations using quotients of formal solutions and integral bases. J. Symbolic Comput., 83:254-271, 2017.

[44] M. Kauers, C. Koutschan, and D. Zeilberger. Proof of Ira Gessel's lattice path conjecture. Proc. Natl. Acad. Sci. USA, 106(28):11502-11505, 2009.

[45] M. Kauers and D. Zeilberger. The quasi-holonomic ansatz and restricted lattice walks. J. Difference Equ. Appl., 14(10-11):1119-1126, 2008.

[46] C. Koutschan. A fast approach to creative telescoping. Math. Comput. Sci., $4(2-3): 259-266,2010$

[47] C. Koutschan. Creative telescoping for holonomic functions. In Computer algebra in quantum field theory, Texts Monogr. Symbol. Comput., pages 171-194. Springer, Vienna, 2013

[48] C. Krattenthaler. Lattice path enumeration. In Handbook of enumerative combinatorics, Discrete Math. Appl., pages 589-678. CRC Press, 2015.

[49] G. Kreweras. Sur une classe de problèmes de dénombrement liés au treillis des partitions des entiers. Cahiers du B.U.R.O., 6:9-107, 1965.

[50] G. Kreweras and H. Niederhausen. Solution of an enumerative problem connected with lattice paths. European J. Combin., 2(1):55-60, 1981

[51] V. J. Kunwar and M. van Hoeij. Second order differential equations with hypergeometric solutions of degree three. In Proc. ISSAC'13 pages 235-242. ACM, 2013.

[52] I. Kurkova and K. Raschel. On the functions counting walks with small steps in the quarter plane. Publ. Math. Inst. Hautes Études Sci., 116(1):69-114, 2012.

[53] A. K. Lenstra, H. W. Lenstra, Jr., and L. Lovász. Factoring polynomials with rational coefficients. Math. Ann., 261(4):515-534, 1982

[54] L. Lipshitz. D-finite power series. J. Algebra, 122(2):353-373, 1989.

[55] S. Melczer and M. Mishna. Singularity analysis via the iterated kernel method. In DMTCS Proceedings of FPSAC'13, Paris, France, pages 481-492, 2013.

[56] S. Melczer and M. C. Wilson. Asymptotics of lattice walks via analytic combinatorics in several variables. In DMTCS Proceedings of FPSAC'16, Vancouver, Canada, pages 863-874, 2016.

[57] M. Mishna. Classifying lattice walks restricted to the quarter plane. J. Combin. Theory Ser. A, 116(2):460-477, 2009.

[58] M. Mishna and A. Rechnitzer. Two non-holonomic lattice walks in the quarter plane. Theoret. Comput. Sci., 410(38-40):3616-3630, 2009.

[59] S. G. Mohanty. Lattice path counting and applications. Academic Press, New York, 1979. Probability and Mathematical Statistics.

[60] T. V. Narayana. Lattice path combinatorics with statistical applications, volume 23 of Mathematical Expositions. University of Toronto Press, Ont., 1979.

[61] H. Niederhausen. The ballot problem with three candidates. European J. Combin., 4(2):161-167, 1983.

[62] R. Pemantle and M. C. Wilson. Analytic combinatorics in several variables, volume 140 of Cambridge Studies in Advanced Mathematics. Cambridge University Press, Cambridge, 2013.

[63] D. Popescu. General Néron desingularization and approximation. Nagoya Math. J., 104:85-115, 1986

[64] M. Renault. Lost (and found) in translation: André's actual method and its application to the generalized ballot problem. Amer. Math. Monthly, 115(4):358363, 2008.

[65] B. Salvy. Linear differential equations as a data structure. Found. Comput. Math., 19(5):1071-1112, 2019.

[66] H. A. Schwarz. Über diejenigen Fälle, in welchen die Gaußische hypergeometrische Reihe einer algebraische Funktion ihres vierten Elementes darstellt. J. Reine Angew. Math., 75:292-335, 1873.

[67] N. J. A. Sloane. The on-line encyclopedia of integer sequences, https://oeis.org.

68] R. P. Stanley. Differentiably finite power series. European J. Combin., 1(2):175$188,1980$.

[69] M. van Hoeij. Factorization of differential operators with rational functions coefficients. J. Symbolic Comput., 24(5):537-561, 1997.

[70] H. S. Wilf and D. Zeilberger. An algorithmic proof theory for hypergeometric (ordinary and " $q$ ") multisum/integral identities. Invent. Math., 108(3):575-633, 1992.

[71] D. Zeilberger. The method of creative telescoping. J. Symbolic Comput., 11(3):195204, 1991. 